\title{
Could Sotrovimab, a monoclonal antibody, be helpful in COVID- 19 symptoms in cats and ferrets?
}

Serkan Sayıner ${ }^{1,2, *}$ and Ahmet Özer Şehirli ${ }^{3}$

${ }^{1}$ Department of Biochemistry, Faculty of Veterinary Medicine, Near East University, 99138 Nicosia, Northern Cyprus, Mersin 10, Turkey ${ }^{2}$ Diagnostic Laboratory, Animal Hospital, Near East University, 99138 Nicosia, Northern Cyprus, Mersin 10, Turkey

${ }^{3}$ Department of Pharmacology, Faculty of Dentistry, Near East University, 99138 Nicosia, Northern Cyprus, Mersin 10, Turkey

*Corresponding author: Serkan Sayıner, Department of Biochemistry, Faculty of Veterinary Medicine, Near East University, 99138 Nicosia, North Cyprus, Mersin 10, Turkey

Received date: 11 December, 2021 | Accepted date: 24 December, 2021 | Published date: 27 December, 2021

Citation: Sayıner S, Şehirli AO. (2021) Could Sotrovimab, a monoclonal antibody, be helpful in COVID-19 symptoms in cats and ferrets? J Clin Vet Res 1(2): doi https://doi.org/10.54289/JCVR2100106

Copyright: () 2021 Sayıner S, Şehirli AO. This is an open-access article distributed under the terms of the Creative Commons Attribution License, which permits unrestricted use, distribution, and reproduction in any medium, provided the original author and source are credited.

\section{Abstract}

Although the types of coronaviruses seen in animals differ, recent studies have also shown that they are affected by COVID-19, known as SARS-CoV-2. The impact of COVID-19 on animals is a factor that should be followed carefully, especially since different variants appear in humans every day and this disease is transmitted from human to animal. Thanks to vaccination, animals are less affected by different types of coronaviruses. Cats and ferrets are especially affected much more in COVID-19, causing damage to the lungs and other organs. Recently, it has been shown that the use of monoclonal antibodies, especially in the early stages of COVID-19, by people with chronic diseases, positively affects the course of the disease, reduces the frequency of hospitalization and the possibility of falling into intensive care. Sotrovimab is a pan-sarbecovirus monoclonal antibody, and 12-13 studies to date have shown that individuals with chronic disease are less affected when given in the early stages of the disease when the symptoms are mild. We also think that if especially old cats and ferrets are treated with Sotrovimab in the early stages of the disease when they contract COVID-19, it will positively affect the prognosis of the disease.

Keywords: Cats, COVID-19, Ferrets, SARS-CoV-2, Sotrovimab, Vaccination

Abbreviations: OIE: World Organisation for Animal Health, BCoV: Bovine Respiratory Coronavirus, CCov: Canine Coronavirus, FIPV: Feline Infectious Peritonitis Virus, TGEV: Transmissible Gastroenteritis Coronavirus, FCoV: Feline Coronavirus

\section{Introduction:}

SARS-CoV-2, known as COVID-19, which affects the world in both health and economic fields, affects all animals, mostly cats and ferrets, according to the World Organization of Animal Health (OIE) data [1-3]. Although the rate of morbidity and mortality in humans is increasing day by day, studies on this subject in animals are limited. However, although different types of coronaviruses occur in animals, protection is provided in animals thanks to vaccination $[\mathbf{4 , 5}$. It has been determined that SARS CoV-2 is transmitted from humans to animals $[\mathbf{6 , 7 ]}$. It has been suggested that especially the lungs and neuronal tissues of animals may be affected and may cause permanent damage $[\mathbf{8 , 9}$ ]. Therefore, there is a need to administer new agents with low side effects and strong efficacy to animals during the disease, together with vaccines. Recent studies have shown that treatments with 
monoclonal antibodies in humans reduce the frequency of hospitalization $[\mathbf{1 0}, \mathbf{1 1}]$. In particular, the emergence of different variants and the consequences of these variants on animals is an important issue that needs to be investigated. Therefore, examining and evaluating the effects of possible therapeutic agents on animals is an undeniable fact in terms of veterinary medicine. The most effective solution is the development of a monoclonal antibody that neutralizes SARS-CoV-2 by targeting an evolutionarily conserved epitope that lies outside the rapidly evolving receptor-binding motif [12-14]. This antibody should have a high barrier to resistance and the receptor-binding motif can be combined with targeted antibodies as needed to further raise the barrier against resistance.

Sotrovimab (VIR-7831) is a monoclonal antibody prepared to neutralize sarbecoviruses such as SARS-CoV-1 and SARSCoV-2 $[\mathbf{1 5}, \mathbf{1 6}]$. In vitro studies have shown that it is effective against all variants that have emerged so far. Clinical studies on humans have also shown that it can reduce the frequency of hospitalization of people with chronic diseases $[\mathbf{1 0 , 1 6 , 1 7 ]}$. Therefore, in the light of these findings, we think that Sotrovimab may be beneficial for both ferrets and cats.

\section{Animal CoV Infections}

There are different types of coronaviruses on animals, and they also affect domestic, wild and farm animals and cause mortality and morbidity that negatively affect life. There are different types such as the type seen in cattle $(\mathrm{BCoV})$, pigs (TGEV, PDEV), dogs (CCoV), cats (FCoV) [18]. Although gastrointestinal symptoms can be seen in cattle, pigs and dogs, it has been suggested that it can affect almost all organs in cats [feline infectious peritonitis virus (FIPV)] and ferrets $[19,20]$. In this disease, the cytokine storm together with the activation of macrophages adversely affects the lungs as in humans. It is thought that especially old and diseased cats will be more affected $[\mathbf{2 1}, \mathbf{2 2}]$. In this context, the importance of agents that modify the immune system is increasing day by day in both humans and animals. Therefore, the necessity of monoclonal antibody treatment with antiviral drug in the first period of the disease is inevitable.

SARS-CoV-2 can be transmitted from person to person through the respiratory tract, as well as from person to animal through the ACE-2 and TMPRSS-2 enzymes [23, 24]. It has also been reported in animals in many countries $[3,8]$. Especially in experimental studies, it is thought that cats and ferrets may be sensitive to all variants of COVID-19, similar to humans, and may affect all tissues with macrophage activation and cytokine storm [25-27].

\section{COVID-19 and Sotrovimab}

As with viral infections, vaccines are required for eradication of SARS-CoV-2 [28]. Although prophylaxis is provided by vaccination, different approaches are required for the radical treatment of the disease. The importance of monoclonal antibodies has been increasing recently to positively affect the prognosis of the disease and reduce morbidity and mortality in SARS-CoV-2 [29]. It was established that the recently found Sotrovimab not only affects ACE-2, but also the enzymes and proteins that the virus invades, such as TMPRSS-2, CD-147 [15-17]. Thus, Sotrovimab can also inhibit cytokine storm with free oxygen radicals that occur with replication after SARS-CoV-2 invasion. Studies in animals have shown that Sotrovimab neutralizes virus binding [16].

In conclusion, it is thought that the administration of Sotrovimab, which is a monoclonal antibody to be made in the first place where SARS-CoV-2 in animals affects cats and ferrets the most, together with antiviral treatment may positively affect the prognosis of the disease and reduce mortality and morbidity.

Conflict of interest: Authors declare no conflict of interest.

\section{References:}

1. Schlottau K, Rissmann M, Graaf A, Schon J, Sehl J, et al. (2020) SARS-CoV-2 in fruit bats, ferrets, pigs, and chickens: an experimental transmission study. The Lancet Microbe 1: e218-e225.

2. Halfmann PJ, Hatta M, Chiba S, Maemura T, Fan S, et al. (2020) Transmission of SARS-CoV-2 in Domestic Cats. New England J Medic. 383: 592-594.

3. Curukoglu A, Ergoren MC, Ozgencil FE, Sayiner S, Ince ME, et al. (2021) First direct human-to-cat transmission of the SARS-CoV-2 B.1.1.7 variant. Aust Veter J. 99: 482-488. 
4. Saif LJ. (2004) Animal coronavirus vaccines: lessons for SARS. Developments in Biologicals 119: 129-140.

5. Sharun K, Tiwari R, Saied AA, Dhama K. (2021) SARSCoV-2 vaccine for domestic and captive animals: An effort to counter COVID-19 pandemic at the human-animal interface. Vaccine. 39: 7119-7122.

6. Prince T, Smith SL, Radford AD, Solomon T, Hughes GL, et al. (2021) SARS-CoV-2 Infections in Animals: Reservoirs for Reverse Zoonosis and Models for Study. Viruses 13(3): 494.

7. Bashor L, Gagne RB, Bosco-Lauth AM, Bowen RA, Stenglein M, et al. (2021) SARS-CoV-2 evolution in animals suggests mechanisms for rapid variant selection. Proc Natl Acad Sci U S A. 118(44): e2105253118.

8. Perisé-Barrios AJ, Tomeo-Martín BD, Gómez-Ochoa P, Delgado-Bonet P, Plaza P, et al. (2021) Humoral responses to SARS-CoV-2 by healthy and sick dogs during the COVID-19 pandemic in Spain. Veter Research. 52: 22.

9. Stevanovic V, Tabain I, Vilibic-Cavlek T, Maljkovic MM, Beniv I, et al. (2021) The emergence of sars-cov-2 within the dog population in croatia: Host factors and clinical outcome. Viruses 13(8): 1430.

10. Kreuzberger N, Hirsch C, Chai KL, Tomlison E, Khosravi $\mathrm{Z}$, et al. (2021) SARS-CoV-2-neutralising monoclonal antibodies for treatment of COVID-19. Cochrane Database of Systematic Reviews.

11. Rubin EJ, Baden LR, Morrissey S. (2021) Audio Interview: A New Monoclonal Antibody for Covid-19 and Potential Vaccination for Children. N Engl J Med. 385: e74.

12. Zost SJ, Gilchuk P, Case JB, Binshtein E, Chen RE, et al. (2020) Potently neutralizing and protective human antibodies against SARS-CoV-2. Nature 584: 443-449.

13. Kramer KJ, Johnson N v., Shiakolas AR, Suryadevara N, Sivakumar P, et al. (2021) Potent neutralization of SARSCoV-2 variants of concern by an antibody with an uncommon genetic signature and structural mode of spike recognition. Cell Reports 37: 109784.

14. Wang P, Casner RG, Nair MS, Yu J, Guo Y, et al. (2021) A monoclonal antibody that neutralizes SARS-CoV-2 variants, SARS-CoV, and other sarbecoviruses. bioRxiv : the preprint server for biology.
15. (2021) New drug: Sotrovimab for COVID-19. Australian Prescriber 44:175

16. Gupta A, Gonzalez-Rojas Y, Juarez E, Casal MC, Moya J, et al. (2021) Early Treatment for Covid-19 with SARS-CoV2 Neutralizing Antibody Sotrovimab. N Engl J Med. 385:1941-1950.

17. Mahase E. (2021) Covid-19: UK approves monoclonal antibody sotrovimab for over 12s at high risk. BMJ 375: n2990.

18. Zappulli V, Ferro S, Bonsembiante F, et al. (2020) Pathology of Coronavirus Infections: A Review of Lesions in Animals in the One-Health Perspective. Animals 10(12): 2377.

19. Felten S, Hartmann K. (2019) Diagnosis of Feline Infectious Peritonitis: A Review of the Current Literature. Viruses 11(11): 1068 .

20. Murray J, Kiupel M, Maes RK. (2010) Ferret CoronavirusAssociated Diseases. Veterinary Clinics of North America: Exotic Animal Practice 13:543-560.

21. Cloutier M, Nandi M, Ihsan AU, Chamard HA, Ilangumaran S, et al. (2020) ADE and hyperinflammation in SARS-CoV2 infection comparison with dengue hemorrhagic fever and feline infectious peritonitis. Cytokine 136:155256.

22. Carvallo FR, Martins M, Joshi LR, Caserta LC, Mitchell PK, et al. (2021) Severe SARS-CoV-2 Infection in a Cat with Hypertrophic Cardiomyopathy. Viruses 13:1510.

23. Cevik M, Kuppalli K, Kindrachuk J, Peiris M. (2020) Virology, transmission, and pathogenesis of SARS-CoV-2. BMJ 371: m3862.

24. Zang R, Gomez Castro MF, McCune BT, et al. (2020) TMPRSS2 and TMPRSS4 promote SARS-CoV-2 infection of human small intestinal enterocytes. Science immunology.

25. Bonilauri P, Rugna G. (2021) Animal coronaviruses and SARS-CoV-2 in animals, what do we actually know? Life 11:1-17.

26. Tiwari R, Dhama K, Sharun K, Iqbal Yatoo M, Malik YS, et al. (2020) COVID-19: animals, veterinary and zoonotic links. The veterinary quarterly 40(1): 169-182.

27. Muñoz-Fontela C, Dowling WE, Funnell SGP, et al. (2020) Animal models for COVID-19. Nature 586: 509-515. 
28. Walsh EE, Frenck RWJ, Falsey AR, Kitchin N, Absalon J, et al. (2020) Safety and Immunogenicity of Two RNABased Covid-19 Vaccine Candidates. The New England Journal of Medicine 383: 2439-2450.
29. Tuccori M, Ferraro S, Convertino I, Cappello E, Valdiserra G, et al. (2020) Anti-SARS-CoV-2 neutralizing monoclonal antibodies: clinical pipeline. mAbs. 12(1). 